This item was submitted to Loughborough's Research Repository by the author.

Items in Figshare are protected by copyright, with all rights reserved, unless otherwise indicated.

\title{
Measurement of a biomimetic antenna in the shape of a bat's ear
}

PLEASE CITE THE PUBLISHED VERSION

PUBLISHER

(C) IEEE

VERSION

VoR (Version of Record)

LICENCE

CC BY-NC-ND 4.0

REPOSITORY RECORD

Farmer, G., James A. Flint, Gordon Leonard, and S.A. Dible. 2019. "Measurement of a Biomimetic Antenna in the Shape of a Bat's Ear". figshare. https://hdl.handle.net/2134/6172. 
This item was submitted to Loughborough's Institutional Repository (https://dspace.lboro.ac.uk/) by the author and is made available under the following Creative Commons Licence conditions.

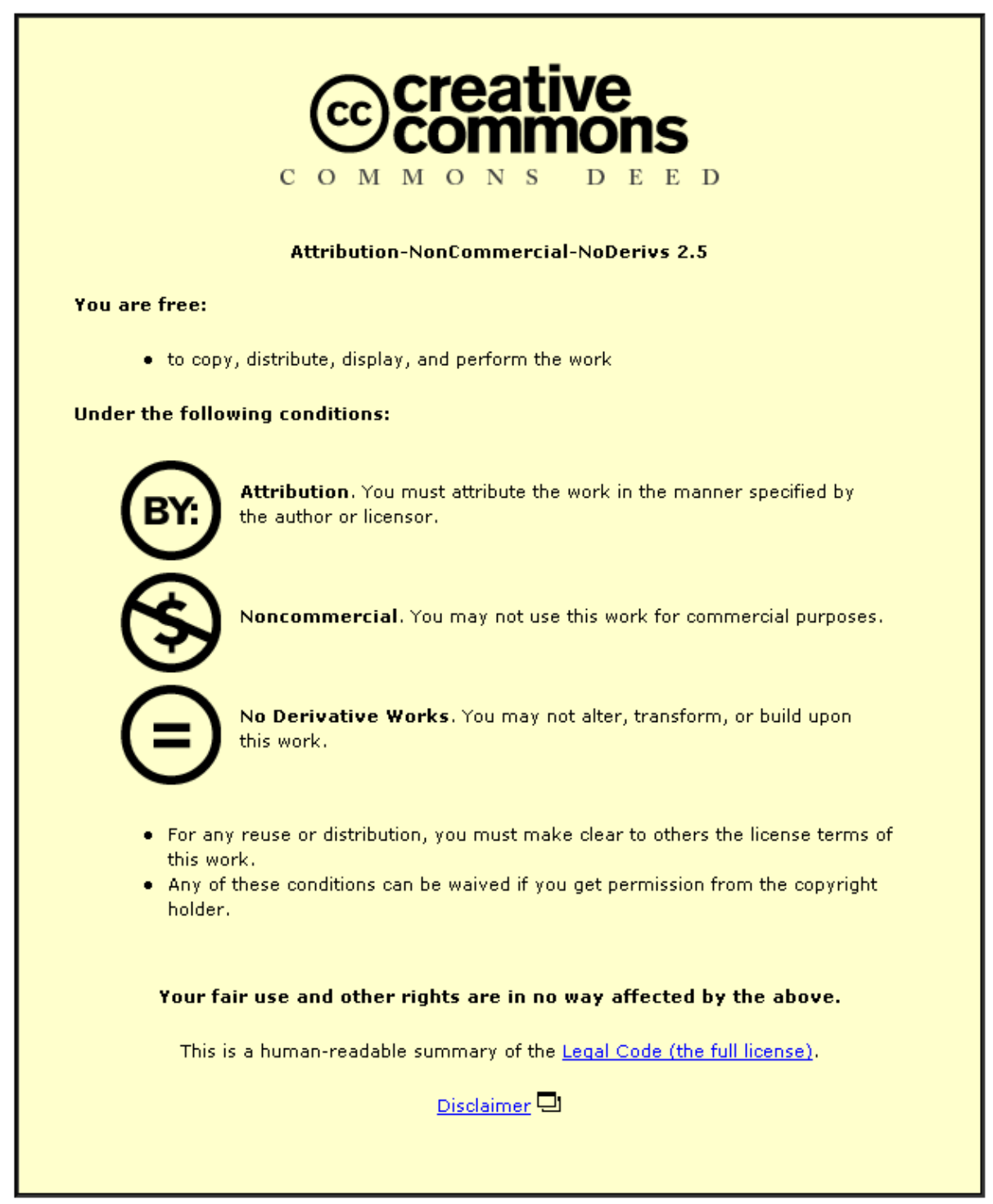

For the full text of this licence, please go to: http://creativecommons.org/licenses/by-nc-nd/2.5/ 


\title{
MEASUREMENT OF A BIOMIMETIC ANTENNA IN THE SHAPE OF A BAT'S EAR
}

\author{
G Farmer, J A Flint, G Leonard, S A Dible \\ Department of Electronic and Electrical Engineering, Loughborough University, \\ Ashby Road, Loughborough, Leicestershire,UK, Email: james.flint@ieee.org
}

\begin{abstract}
This paper presents a series of measurements of a novel antenna that physically resembles the ear of a bat. The antenna consists of a circular ground plane with a central monopole element. An equilateral triangular conducting plate is curved around the ground so that the base of the triangle is electrically connected to the perimeter of the circle and is of the same length. The input characteristic is reminiscent of a simple monopole above a circular ground, providing there are a sufficient number of modes in the triangular plate at the frequency of interest. In contrast to the plain monopole, certain frequencies yield a high gain and a radiation pattern with low side lobes. Measurements presented in this paper suggest that the antenna performance is broadly comparable with its acoustic analogue, although there are differences between the acoustic and electromagnetic implementation which have yet to be resolved.
\end{abstract}

\section{INTRODUCTION}

Bats (Chiroptera) are unique in the animal kingdom being the only mammals to have true wings and powered flight. Bats of the suborder microchiroptera (so-called Microbats) rely heavily on echolocation to navigate and hunt at night. They transmit sound from the mouth and/or nose which reflects from their surroundings or prey. The returning echo is detected via the bat's external ears or pinnae. These pinnae are complex shapes which are very sensitive and highly optimised by evolution to their task. They are able to combine this sensory capability with high maneuverability and can achieve impressive interception and navigation tasks. For this reason, and because in many aspects bat sonar appears to outperform current human-made systems, there has been a lot of interest in the acoustics research community in fully understanding and replicating these pinna shapes $[1,2,3]$. In one of the latest developments, a simplified bat ear (electromagnetic) antenna was proposed and initial computer modeling work suggested that the shape would make a viable electromagnetic transmitting/receiving device [4]. Some earlier work (e.g. [5]) has explored bat's ears as a possible antenna design, however to our knowledge the work presented in this paper is the first attempt to systematically produce and evaluate a bat-ear antenna via a combination of both acoustic and electromagnetic simulations.

The purpose of the current paper is to present a series of measurement results on this device and to further explain the operation of the antenna. Results are also presented which show the receive pattern in the acoustic domain for a specific bat ear shape. The species Rhinolophus Rouxii (the Rufous Horseshoe bat) has been investigated via acoustic modelling and measurement, and makes a useful subject for comparison with the antenna.

\section{ANTENNA GEOMETRY}

The basic antenna design is shown in Fig. 1.
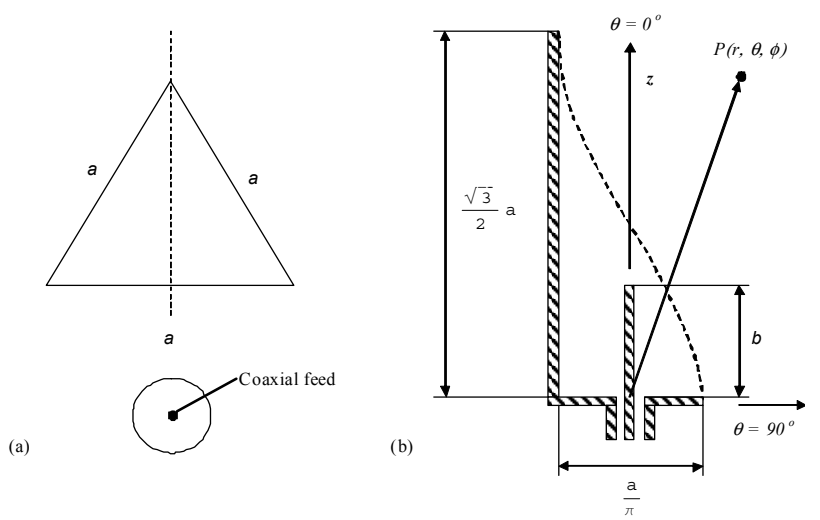

(c)

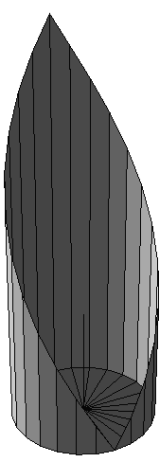

Figure 1. (a) flat component parts of the bat ear antenna. (b) cutaway view (c) $3 D$ projection.

Note that the construction is extremely simple; it 
consisting only of a triangular metallic plate and a base disc. The presence of the curved triangular plate governs the electromagnetic behaviour of the device. When the triangle/disc structure is driven by the centrally-positioned monopole element it excites a subset of the modes of the triangle. It has already been established that some of these modes are quite strongly excited by this feeding method [4].

The equation for calculating the triangular resonant frequencies is given in Eq. 1.

$$
f_{m n l}=\frac{2 c}{3 a}\left(m^{2}+m n+n^{2}\right)^{1 / 2}
$$

Where $c$ is the speed of light, $a$ is the side length of the triangle and $m, n$ are mode index numbers. Further details about resonance in triangular structures can be found in $[6,7,8]$.

The synthetic bat's ear used in the measurement campaign was constructed from thin tinned steel. A photograph of the finished component is shown in Fig. 2.

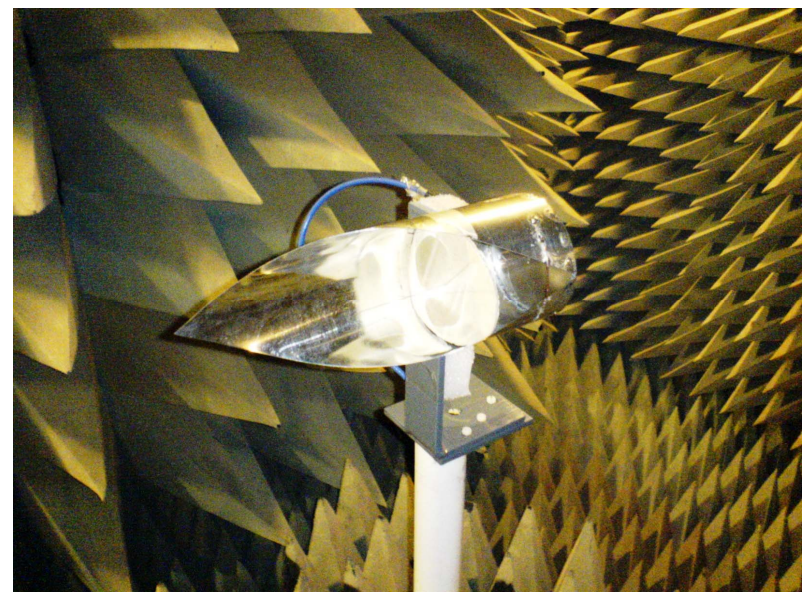

Figure 2. Artificial 'bat's ear' antenna undergoing measurement in a fully-anechoic chamber.

The connection between the triangular patch and the base was soldered to ensure a good conductive path. In addition a pair of non-conducting support struts were introduced in order to maintain an approximately cylindrical profile along the length. An SMA connector was attached to the centre of the base with the outer conductor soldered to the antenna body and with the inner conductor protruding into the antenna. A hole was drilled to permit this, and the diameter was selected in order to minimise the effects of any changes in the characteristic impedance. The feeding monopole was attached so that it was coaxial to the antenna's cylindrical profile. For the longer monopoles it was necessary to introduce a support disc of non-conducting material into the device (visible in Fig. 2) and to pass the monopole element through it. This was important to minimise sagging in the element.

\section{ACOUSTIC MEASUREMENTS}

A combination of both numerical and measurement techniques were used in order to evaluate the original biological structure. The source data for both the measurement and modelling was a very high resolution CT (computed tomography, X-ray) data set acquired from a post-mortem specimen of Rhinolophus Rouxii. Projections from this data set are depicted inset in Fig. 3.

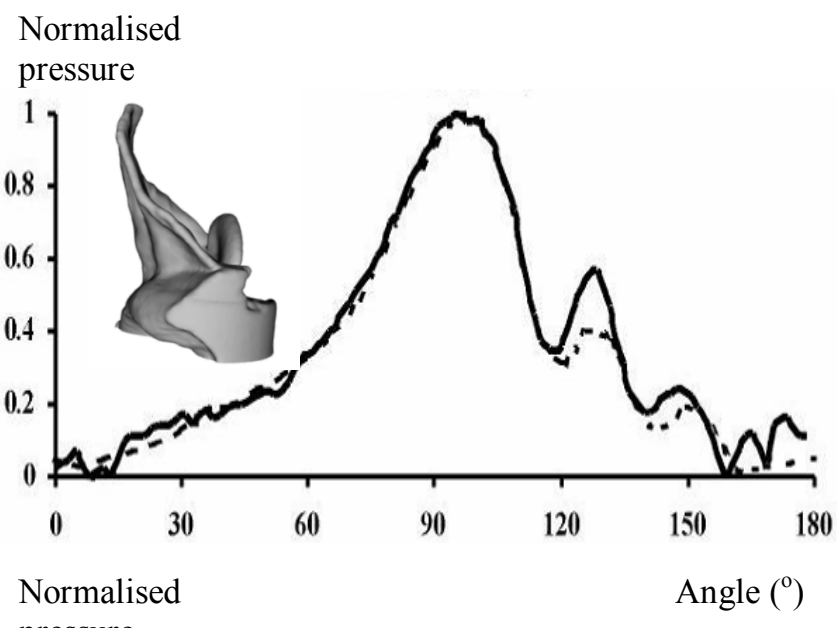
pressure

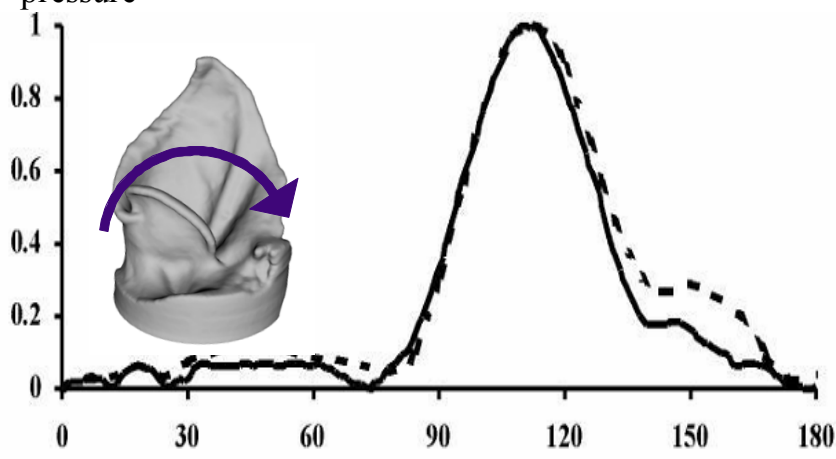

Angle $\left({ }^{\circ}\right)$

Figure 3. Far field radiation patterns of Rhinolophus Rouxii at $80 \mathrm{kHz}$ (measured result shown with a solid line, modelled results shown dotted)

The CT data was post-processed for numerical modelling by down sampling and then passing the resultant analysis mesh to an in-house simulator package based on the TLM method [9]. A time-domain TLM method was selected as it is able to simulate a very wide frequency band, has high spectral resolution, and is in theory able to account for the effects of diffraction. The near field results generated by the simulation software were subsequently transformed via 
a numerical extrapolation technique to the far field. In parallel with this activity, a three-dimensional object was constructed from the source data set by means of a stereo-lithography technique. The manufacturing material was a specialised prototyping plastic for which very little acoustic data was available, however it was assumed that the material would be a reasonable reflector of sound at the frequency being measured.

The specimen manufactured was attached to a speciallymade polar measurement rig and fed via an ultrasonic transducer and a thin tube. The results of the measurements made at $80 \mathrm{kHz}$ are shown in Fig. 3. This frequency was selected because the bat in question is known to make use a prolonged continuous frequency component in its emissions at that particular frequency. It is therefore reasonable to expect that good (possibly optimal) performance would be achieved.

The patterns clearly indicate a major lobe which is tilted away from the centre axis. In the top image it is also clear that the pattern is asymmetric with the majority of the side lobes concentrated on one side of the major lobe. The modelled results from TLM are in good agreement with the measurements made.

\section{ELECTROMAGNETIC MEASUREMENTS}

\subsection{Input characteristics}

Measurements and numerical simulations were carried out in order to determine the input characteristics (return loss) and to assess the directivity. A computer model employing a commercial TLM simulator was used in order to study parameters $a$ and $b$ (See Fig. 1 for details of the parameterisation). The physical prototype depicted in Fig. 2 was fabricated with $a=260 \mathrm{~mm}$ and investigated with different values of $b$ in the range $0<b / a \leq \sqrt{3} / 2$. Note that $b / a=\sqrt{3} / 2$ corresponds to the case where the monopole length is equal to the height of the triangle with side length $a$. In the computer model both the monopole and the triangular plate were considered as thin structures.

For the cases $b / a=0.5$ and $b / a=0.25$ the return losses obtained at the antenna terminals are shown in Figs. 4 and 5 respectively. In each case the measured result from the device is compared with a TLM simulation (dotted), and also with a simulation of the circular ground plane by itself (thin line, dashed).

The modelled and measured results agree reasonably well in terms of frequency and amplitude. At some frequencies a low amplitude resonance appears in the plot which is not a natural frequency of the monopole above ground. An example of this can be found in
Fig. 5 at approximately $7 \mathrm{GHz}$. This can be attributed to a strong resonance in the triangular plate and is predicted accurately by Eq. 1. These triangle-mode frequencies are indicated on the graphs by the triangular markers on the $0 \mathrm{~dB}$ axis. Despite these minor differences, the response is still broadly similar to the plain monopole and therefore the design process to achieve a good input match is quite straightforward.

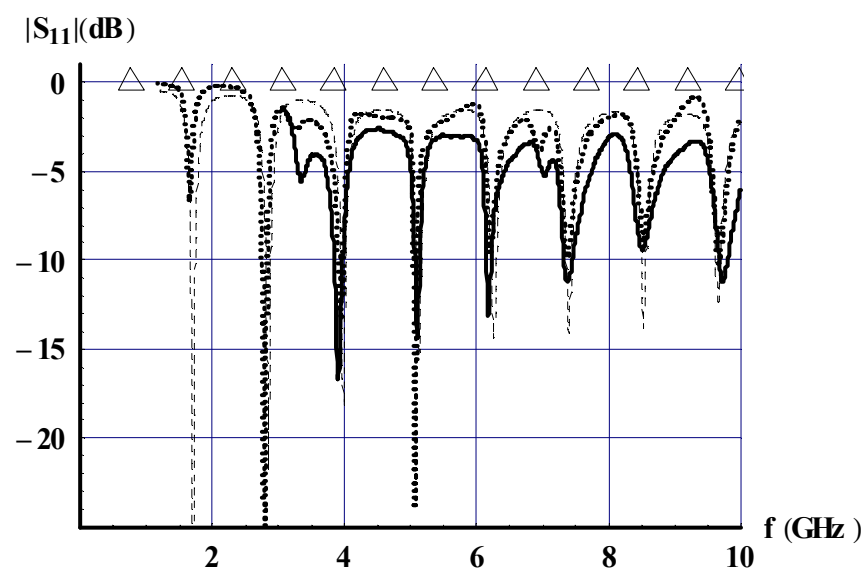

Figure 4. Measured and simulated return loss at the antenna input for $b / a=0.50$.

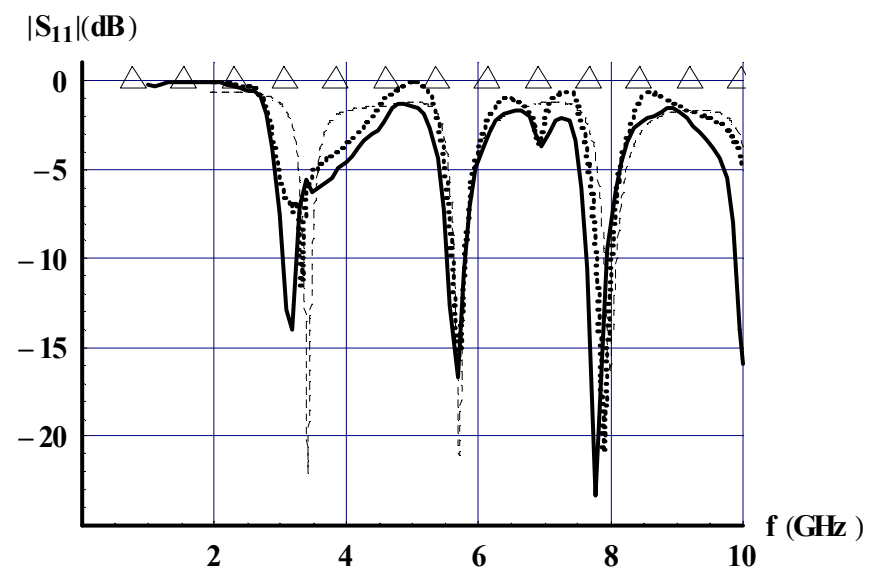

Figure 5. Measured and simulated return loss at the antenna input for $b / a=0.25$

\subsection{Radiation patterns}

The radiation patterns were measured and modelled for a number of frequencies and monopole lengths. Each of the patterns modelled had a particular characteristic shape governed by the modal structure of the triangle. Since there are too many of these to report, a sample of the results is presented in Figs. 6 and 7.

Fig. 6 shows the patterns relating to two strong modes found via the s-parameter measurements. It is clear that 
both patterns have a major lobe which points slightly away from the axis. In (b), however there is a much higher gain compared to (a). When these patterns are compared qualitatively with those from the bat in the acoustic domain, there is a broad similarity. One noticeable difference is the lack of some of the fine side lobe structure found in the acoustic case. Indeed, some differences were expected because this antenna is inherently simpler than the object that it is aiming to mimic. In addition, the biological specimen used was a dead bat and therefore it is impossible to be sure that the shape had not changed prior to $3 \mathrm{D}$ scanning. In the living animal the shape of the ear is retained by the surrounding tissue including muscles which are able to change its position.

(a)

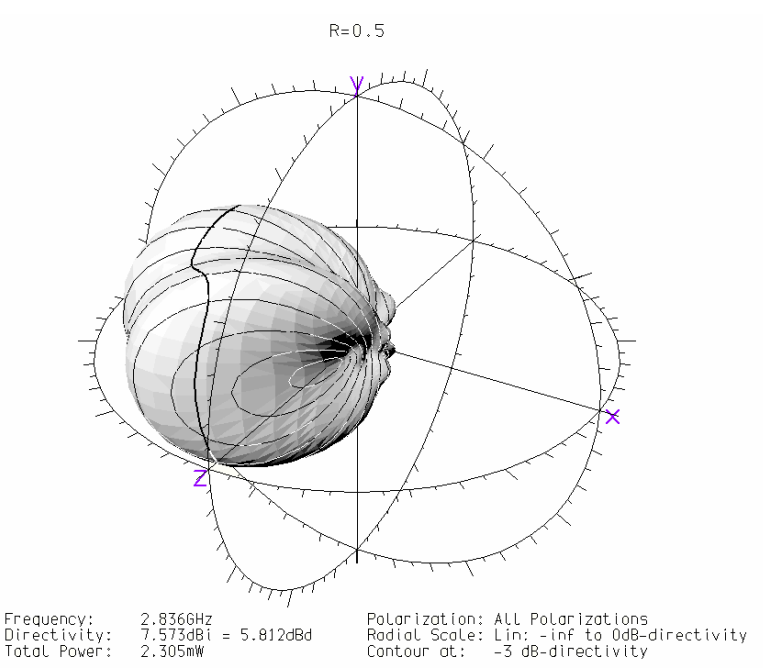

(b)

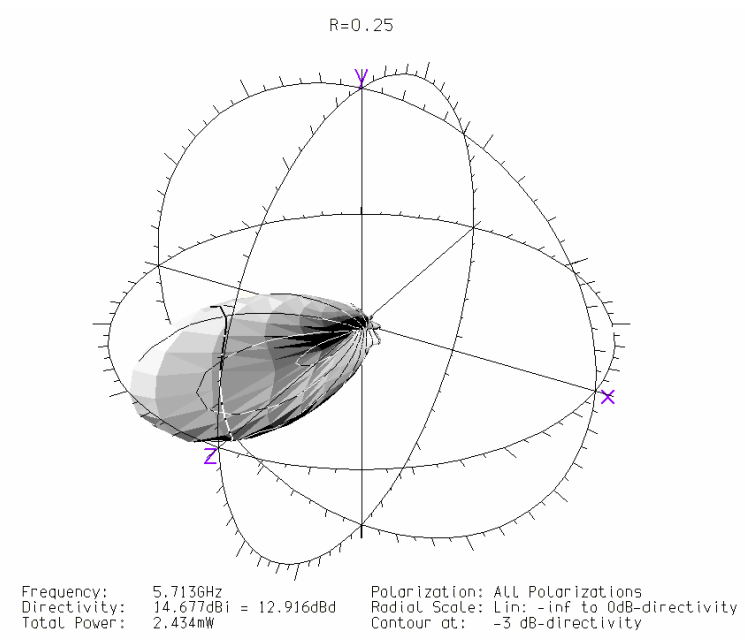

Figure 6. 3D radiation patterns modelled using TLM. (a) for b/a $=0.5$ at $2.836 \mathrm{GHz}$ and (b) for $b / a=0.25 a t$ $5.713 \mathrm{GHz}$
In order to validate the numerical models a number of measurements were made. One area of concern is the possibility of diffraction being an issue in the model. Fig. 7 shows cuts from the radiation patterns in Fig. 6 . Several examples were compared and in each case the numerical result gave excellent or very good agreement with the modelled result, however it was not possible to examine all possible operating modes.

Given this outcome, plus the previous results in acoustics there was no evidence that diffraction is a serious problem for antennas of this type, however a more extensive study would be desirable. Another issue is the presence of cross-polar components, which again requires further investigation.

\section{CONCLUSION}

In this paper a set of measurements has been presented which demonstrate the viability of a biomimetic antenna for electromagnetic applications. Such a device has high directivity and a cylindrical physical profile which could be exploited in applications where the antenna is to be concealed. The input characteristic of the device appears to be dominated by the monopole-above-ground whereas the patterns are mainly determined by a subset of the modes of the triangular plate. The research on this structure currently ignores some of the more detailed features of bat ears such as the tragus found on the front edge of the ear, and also the analogy between the feeding of the bat ear and the antenna is weak. The bat uses a small pipe which can efficiently carry acoustic energy to the inner ear, whereas an electromagnetic waveguide with the same dimensions would be operating below its cut off frequency. This is the main reason for selecting a monopole feed in the current design. Future research will focus on applications for this device and will aim to improve the analogy to the biological world. 
(a)

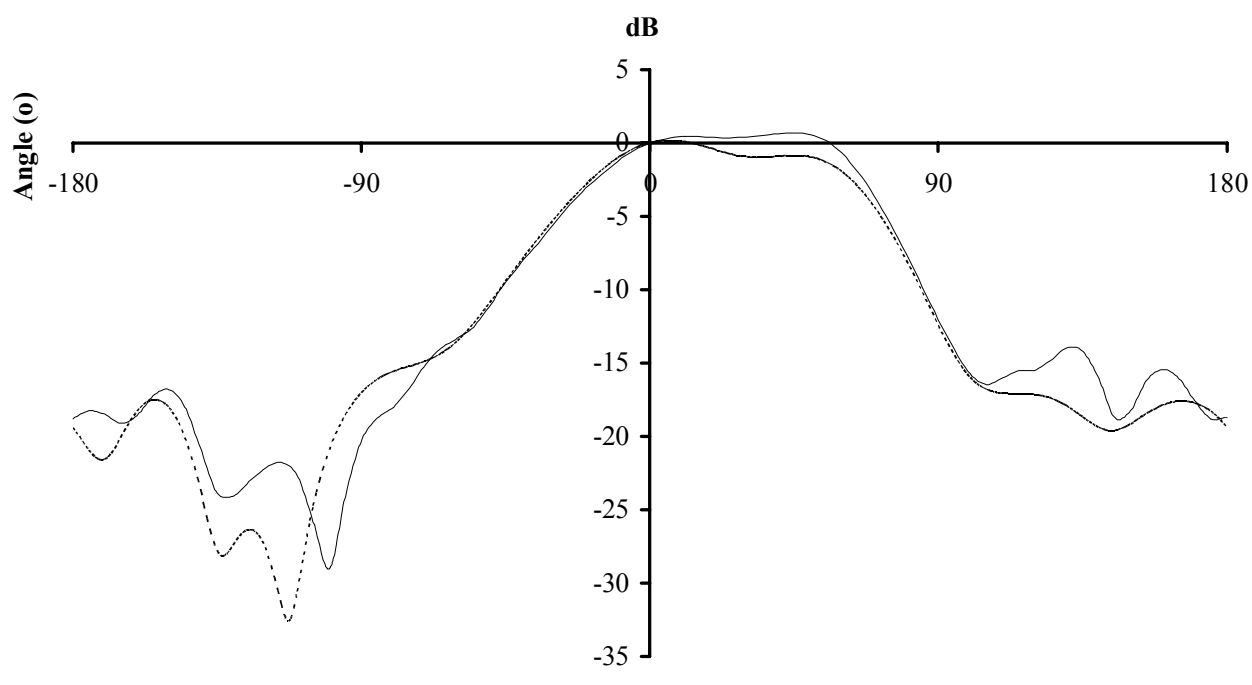

(b)

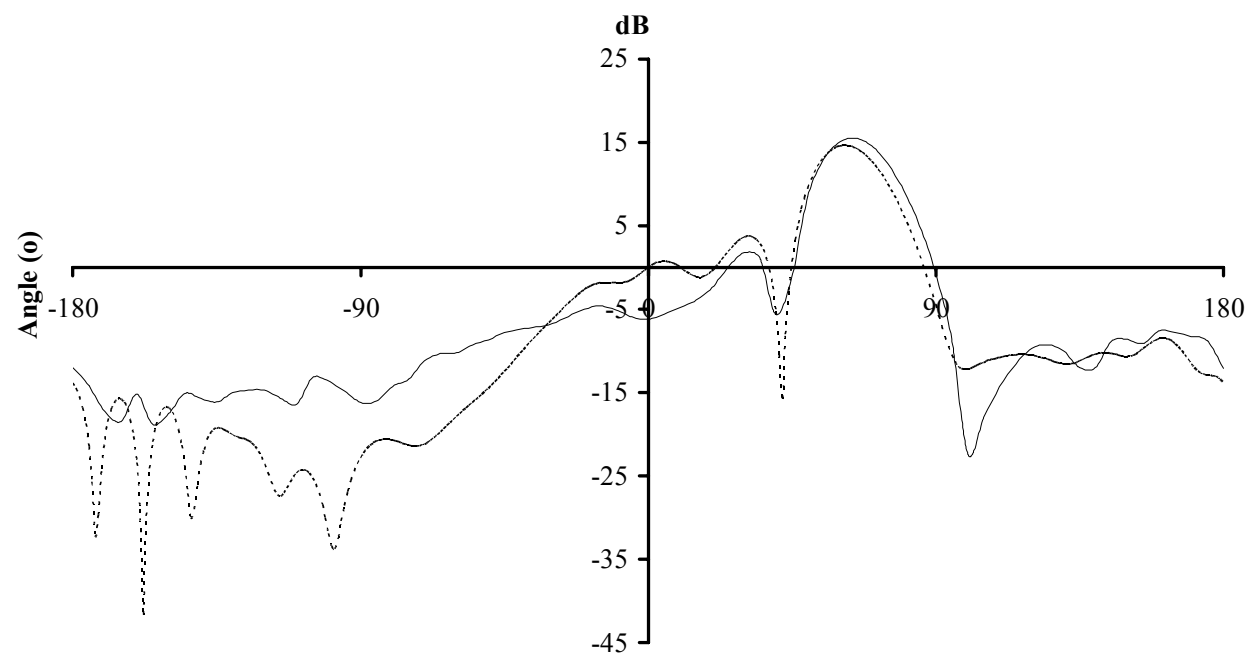

Figure 7. Copolar cut taken on the E-plane. (a) for b/a $=0.5$ at $2.836 \mathrm{GHz}$ and (b) for b/a $=0.25$ at $5.713 \mathrm{GHz}$. The modelled results are shown with a dotted line.

\section{ACKNOWLEDGEMENTS}

The authors were financially supported by The Royal Society, EPSRC, (both UK) and The Department of Electronic and Electrical Engineering at Loughborough University. They wish to thank Flomerics Group for access to the Microstripes TLM software, and Rolf Muller for providing the source $\mathrm{CT}$ data for the acoustic evaluation.

\section{REFERENCES}

1. R. Muller and J.C. Hallam "Knowledge mining for biomimetic smart antenna shapes," Robotics and Autonomous Systems, vol. 50, no. 4, pp. 131-145, 2005.

2. J. Thomas, C. Moss and M. Vater, Ecolocation in Bats and Dolphins, University of Chicago Press, Chicago, 2004. 
3. G. Leonard, J.A. Flint and R. Müller, Proceedings of the Institute of Acoustics, vol. 26., pt. 6., pp. 2937, 2004.

4. J.A. Flint, "A biomimetic antenna in the shape of a bat's ear," Antenna and Wireless Propagation Letters, vol. 5, pp. 145-147, 2006.

5. B.M. Notaros, B.D. Popovic, J.P. Weem, R.A. Brown and Z. Popovic "Efficient large-domain MoM solutions to electrically large practical EM problems," IEEE Trans. Microw. Theory Tech., vol. 49, no. 1, pp. 151-159, 2001.

6. J. Helszain and D.S. James "Planar triangular resonators with magnetic walls," IEEE Transactions on Microwave Theory and Techniques, vol. 26, no. 2, pp. 95-100, 1978.

7. K.-F. Lee, K.-M. Luk and J.S. Dahele "Characteristics of the equilateral triangular patch antenna," IEEE Transactions on Antennas and Propagation, vol. 36, no. 11, pp. 1510-1518, 1988.

8. B.J. McCartin "Eigenstructure of the equilateral triangle, part I: The Dirichlet problem," SIAM Review, vol. 45, no. 2, pp. 267-287, 2003.

9. P.B. Johns "Symmetrical condensed node for the TLM method," IEEE Transactions on Microwave Theory and Techniques vol. 35, no. 4, pp. 370-377, 1987. 